\title{
CONVIVENCIA E INTERCULTURALIDAD DE LA ACCIÓN EDUCATIVA ESPAÑOLA EN EL EXTERIOR
}

\author{
Cohabitation and interculturalism of the educational spanish in external action
}

Beatriz Sánchez Sánchez

Fecha de recepción: 14/09/2018

Fecha de aceptación: 06/12/2018

RESUMEN: El objetivo del presente artículo es acercar la acción educativa, de los centros españoles en el exterior, desde el enfoque de la convivencia y la interculturalidad en sus aulas. A través de una aproximación al organismo que regula esta acción, se va a presentar una contextualización teórica en los diferentes tipos de centros que el Ministerio de Educación, Cultura y Deporte (MECD) dispone en el exterior y que suponen una apertura internacional de la lengua y la cultura española en contacto con otros sistemas educativos y en otros estados. En diferentes programas y tipos de centros se desarrolla la difusión de la lengua y la cultura española. Para ello, se entra en contacto con otras sociedades, momento en que la convivencia y el ámbito intercultural adquieren su protagonismo. Lo intercultural pretende establecer ese intercambio necesario. "nos permite analizar otras culturas desde sus propios patrones culturales; busca el encuentro y tiene una visión crítica de otras culturas" (Martínez, 2010,50). Como punto de partida se tomará como referencia la Ley Orgánica 2/2006, de 3 de mayo, de Educación que en su preámbulo establece "fomentar la convivencia democrática y el respeto a las diferencias individuales "como uno de sus fines .

PALABRAS CLAVE: Convivencia, Interculturalidad, Educación Internacional, Diversidad Cultural.

ABSTRACT: The objective of this article is to bring the educational action of Spanish centers abroad closer, from the approach of coexistence and interculturality in their classrooms. Through an approach to the body that regulates this action, it will present a theoretical contextualization in the different types of centers that the Ministry of Education, Culture and Sport (MECD) has abroad and that involve an international opening of the language and Spanish culture in contact with other educational systems and in other states. In different programs and types of centers the dissemination of the Spanish language and culture is developed. In order to do so, we come into contact with other societies, at which time coexistence and the intercultural sphere acquire their leading role. The intercultural aims to establish that necessary exchange. "It allows us to analyze other cultures from their own cultural patterns; seeks the encounter and has a critical vision of other cultures "(Martínez, 2010,50). As a starting point, reference will be made to Ley Orgánica 2/2006, de 3 de mayo, on Education, which in its preamble establishes "fostering democratic coexistence and respect for individual differences" as one of its purposes.

KEYWORDS: Coexistence, Interculturality, International Education, Cultural Diversity. 


\section{Introducción}

Como señala el MECD, en su página web, "La convivencia pacífica es la base sobre la que se construye cualquier estado democrático y de derecho". Puntualiza que entre los fines de la Ley Orgánica de Educación (en referencia al artículo 2), no puede haber proceso educativo sin convivencia en las aulas y en las comunidades educativas a las que atienden los centros escolares. (Artículo 2 de la Ley Orgánica de Educación).

La acción educativa de España en el exterior es una competencia que se ejerce dentro del marco general de la acción del Estado. Su ámbito de actuación educativa abarca los niveles correspondientes a las enseñanzas regladas no universitarias en los diferentes países en los que está presente.

\section{Red Educativa del MECD a nivel internacional}

Dentro de la estructura organizativa del Ministerio de Educación, Cultura y Deporte de España se encuentra la Subdirección General de Cooperación Internacional y Promoción Exterior Educativa. Se realiza a través del Ministerio de Educación, Cultura y Deporte en coordinación con el Ministerio de Asuntos Exteriores y de Cooperación y el Ministerio de Hacienda y Administraciones Públicas. Como señala la página web de dicho organismo sus funciones son:

a) La coordinación, impulso y seguimiento de la cooperación internacional y de las relaciones internacionales en materia de educación, formación profesional y universidades, en especial con la Unión Europea y, en particular, la asistencia al Ministro en la preparación de las reuniones del Consejo de Ministros de Educación de la Unión Europea, sin perjuicio de las competencias del Ministerio de Asuntos Exteriores y de Cooperación.

b) La planificación, dirección y gestión de los servicios de educación en el exterior encuadrados en las Consejerías, Agregadurías y Direcciones de Programas existentes en diferentes países, de la Administración educativa en el exterior, y de los centros docentes españoles de titularidad estatal en el extranjero. La elaboración del régimen jurídico de la acción educativa en el exterior, sin perjuicio de las competencias del Ministerio de Asuntos Exteriores y de Cooperación. La autorización de la creación de centros docentes privados en el extranjero.

c) La planificación, dirección y gestión de los programas educativos con el exterior, sin perjuicio de la coordinació con las actuaciones con el exterior que se realicen en el ámbito de la Secretaría General de Universidades.

d) La planificación, en colaboración con la Gerencia de Infraestructuras y Equipamientos de Educación y Cultura, de las necesidades de infraestructuras y equipamientos educativos en el exterior. es con el exterior que se realicen en el ámbito de la Secretaría General de Universidades.

d) La planificación, en colaboración con la Gerencia de Infraestructuras y Equipamientos de Educación y Cultura, de las necesidades de infraestructuras y equipamientos educativos en el exterior.

e) El ejercicio de las competencias del Departamento respecto de los tratados internacionales, 
acuerdos internacionales administrativos y acuerdos no normativos en las materias de educación, formación profesional y universidades, así como el asesoramiento sobre la participación española en organismos internacionales.

f) El desarrollo y gestión de las funciones de la Secretaría de Estado de Educación, Formación Profesional y Universidades como Autoridad Nacional del Programa Erasmus+ u otros programas educativos de la Comisión Europea.

\title{
Tabla 1. Estructura organization de la Subdireccion General de Cooperacion Internacional y Promocion Exterior Educativa dentro del MECD
}

\author{
Secretaria de Estado de Educación / Formación Profesional y Universidades
}

$\downarrow$

Dirección General de Planificación y Gestión Educativa

\section{$\downarrow$}

Subdirección General de Cooperación Internacional y Promoción Exterior Educativa

Fuente: Elaboración propia

\subsection{Objetivos}

Dentro de la política general de expansión de la lengua y la cultura españolas, la acción educativa del Ministerio de Educación, Cultura y Deporte (MECD) en el exterior ha experimentado, en los últimos años un incremento muy considerable que ha llevado consigo un notable esfuerzo en recursos humanos y presupuestarios para mantener e intensificar dicha acción.

Si se toma como punto de partida la Ley Orgánica de Educación 2/2006, de 3 de mayo, se puede observar el alcance que la misma otorga al tema intercultural según los siguientes artículos: "La formación en el respeto y reconocimiento de la pluralidad lingüística y cultural de España y de la interculturalidad como un elemento enriquecedor de la sociedad." (Art. 2)

Pluralidad e interculturalidad son paralelismos en una sociedad basada en el respeto y la diversidad: "el Ministerio de Educación, Cultura y Deporte, en colaboración con las Comunidades Autónomas, favorecerá la movilidad internacional de los docentes, los intercambios puesto a puesto y las estancias en otros países." (Art.103)

Se deja de manifiesto la implicación que se tiene por la formación continua del profesorado y los intercambios culturales como beneficio para el sistema educativo del país: "Corresponde al Gobierno la regulación y la gestión de los centros docentes públicos españoles en el exterior." (Apartado 4 del art.107). Se observa la implicación que adquiere el estado español para garantizar los objetivos que refleja en su normativa de la acción educativa en el exterior. 
Como se podrá observar en este apartado, los centros y programas de la acción educativa en el exterior son muy diversos y una generalización normativa es compleja, en consecuencia, queda de manifiesto su singularidad.

Por su parte, la Ley Orgánica 1/1990, de 3 de octubre, de Ordenación General del Sistema Educativo (LOGSE), en su momento, estableció un nuevo marco por el que el sistema español trataba de dar respuesta a las transformaciones producidas en las últimas décadas y a los desafíos cualitativos derivados de la presencia española en el espacio comunitario europeo. Las novedades que ley citaba requería traducirse, sin duda, en una ordenación de la acción educativa española en el extranjero. Paralelamente, la creación del Instituto Cervantes, según Ley 7/1991, de 21 de marzo, por la que se introdujo un organismo especializado para la difusión del español, vino a asumir parcelas de actuación hasta entonces atendidas por diferentes instancias de la Administración educativa.

Consecuencia de todo ello, se puede afirmar que los objetivos de promoción de la lengua y de la cultura españolas se concretaban en un amplio espectro de programas y actividades desarrollados por la administración del MECD en el exterior, con el apoyo y bajo la coordinación de los Servicios Centrales del Ministerio. Finalmente, con la aprobación del Real Decreto 1027/1993, de 25 junio, se regula la acción educativa española en el exterior y es necesario señalar los objetivos siguientes que se definen en diferentes artículos de la misma, en atención a su relevancia, tanto cuantitativa como cualitativa:

- "Incluir la promoción y organización de las enseñanzas regladas correspondientes a niveles no universitarios del sistema español; Incluir la promoción de los currículos mixtos de contenidos del sistema educativo español y de los propios de otros sistemas educativos; Dirigir dichas modalidades de acción educativa tanto a alumnado de nacionalidad española como extranjera." (Art.2).

- "Promocionar y organizar programas de apoyo en el marco de sistemas educativos extranjeros para la enseñanza de la lengua y cultura españolas; Promocionar programas de apoyo a los intercambios en el ámbito educativo y en el de la investigación; Contribuir a facilitar a los españoles el acceso a la educación en el extranjero; Potenciar la proyección de la educación, la cultura y la investigación españolas en el extranjero.” (Art.3)

- "Contribuir al mantenimiento de vínculos culturales y lingüísticos de los residentes españoles en el exterior; Prestar especial atención a la organización de enseñanzas y actividades dirigidas a residentes españoles escolarizados en niveles no universitarios de los sistemas educativos respectivos" (Art.4)

- "Puesto que la acción educativa en el exterior se integra en el marco más amplio de la promoción y difusión de la cultura y de la lengua españolas de la cooperación internacional, el Ministerio de Educación, Cultura y Deporte coordinará sus actuaciones con las que ejerzan el Ministerio de Asuntos Exteriores y Cooperación, y el Instituto Cervantes."

- "La Subdirección General de Cooperación Internacional desarrolla programas encaminados a atender las necesidades educativas de la población española residente en el extranjero; dar respuesta a las demandas de español como lengua extranjera y contribuir a la promoción de la difusión de la lengua y cultura españolas en el mundo.” (Art.6) 
Sin embargo, tras años de experiencia y la importancia adquirida por los programas desarrollados en determinados países, con demandas permanentes en aumento y con un potencial de crecimiento manifiesto, se detecta la necesidad de una nueva regulación de las Consejerías.

Por ello, el Real Decreto 1138/2002, de 31 de octubre, revisa, reordena y completa la normativa existente hasta el momento en lo referente al número de Consejerías y puestos de agregados y asesores, de tal manera que unos y otros contribuyan a canalizar eficazmente la actividad del Ministerio de Educación, Cultura y Deporte.

Dicho Real Decreto, en su artículo 3, establece como función de las Consejerías de Educación: Promover, dirigir y gestionar las distintas actuaciones en materia de acción educativa previstas en el Real Decreto 1027/1993, de 25 de junio, por el que se regula la acción educativa de España en el exterior:

- Prestar asesoramiento y asistencia técnica, informar y realizar funciones de apoyo a la jefatura y demás órganos de la Misión Diplomática en materia educativa;

- Promover y reforzar las relaciones existentes entre la comunidad educativa española y la del país o países donde desarrolle sus funciones;

- Reunir información sobre las políticas educativas desarrolladas en el ámbito territorial que le corresponda y transmitirla a los órganos oportunos de la Administración española;

- Organizar periódicamente actividades de formación del profesorado en el ámbito territorial de su competencia y, cualquier otra que, debiendo realizarse en el exterior, les sea requerida por los órganos del Departamento en el ejercicio de las competencias que tiene atribuidas en este ámbito.

\section{Oficinas y centros en el exterior}

España mantiene una red de oficinas educativas en más de cuarenta países, en los que está presente actualmente, con los que colabora en Misiones Diplomáticas y en los que las necesidades educativas y científicas requieren su presencia. La red engloba los siguientes oréganos: $\quad 2.1$ Oficinas de Educación

Oficinas de Educación:
a) Consejerías
b) Agregadurías
c) Asesorías
d) Direcciones de programas

Centros de recursos

Centros docentes 
a) Consejerías

$\mathrm{Su}$ objetivo es promover y gestionar las acciones educativas en el extranjero. El Ministerio de Educación, Cultura y Deporte está presente en 17 países a través de las Consejerías de Educación en Alemania, Andorra, Argentina, Australia, Bélgica, Brasil, Bulgaria, China, Estados Unidos, Francia, Italia, Marruecos, Méjico, Polonia, Portugal, Reino Unido y Suiza. Además existen dos consejerías encargadas de las relaciones con organizaciones internacionales:

- La Consejería de Educación en la Delegación Permanente de España ante la OCDE, la UNESCO y el Consejo de Europa.

- La Consejería de Educación y en la Representación Permanente de España ante la Unión Europea.

b) Agregadurías

Las Agregadurías de Educación son oficinas de representación del Ministerio de Educación de España que dependen orgánicamente de las Consejerías y están presentes en ciudades o países donde no existe una Consejería de Educación: Canadá, Colombia, Eslovaquia, Estados Unidos (Los Ángeles, Miami, Nueva York), Hungría, La República Checa, Rumanía y Rusia.

c) Asesorías

La función principal de las asesorías es la de prestar asistencia técnica, pedagógica y lingüística en el ámbito de la difusión de la lengua española a través de: colaboraciones con el profesorado en la adaptación de los currículos a la realidad del país y elaboración de informes sobre la situación de la enseñanza del español dentro del sistema educativo correspondiente. Asimismo, tiene el deber de proporcionar información y asesoramiento sobre el ámbito educativo y cultural español y de colaborar en la elaboración de los programas de español, Para ello, asesora a los responsables educativos del país de destino y adapta los recursos didácticos para la enseñanza del español.

El Ministerio de Educación también destina Asesores Técnicos Docentes a ciudades donde (por lo general) no hay Consejería ni Agregaduría para dirigir Centros de Recursos o estar al frente de oficinas de educación:

Tabla 2. Relación de Asesorías Técnicas Españolas en el exterior.

\begin{tabular}{|l|l|}
\hline \multicolumn{1}{|c|}{ ASESORÍAS } & \multicolumn{1}{|c|}{ UBICACIÓN } \\
\hline AUSTRALIA & Adelaida, Melbourne, Sydney \\
\hline AUSTRIA & Viena \\
\hline BRASIL & $\begin{array}{l}\text { Belém, Belo Horizonte, Cuiabá, Curitiba, Porto Alegre, Río de } \\
\text { Janeiro, Salvador de Bahía y Sao Paulo. }\end{array}$ \\
\hline CANADÁ & Edmonton \\
\hline CHILE & Santiago de Chile \\
\hline
\end{tabular}




\begin{tabular}{|l|l|}
\hline $\begin{array}{l}\text { ESTADOS } \\
\text { UNIDOS }\end{array}$ & $\begin{array}{l}\text { Albuquerque, Atlanta, Austin, Baton Rouge, Bloomington, Boston, } \\
\text { Chicago, Hartford, Houston, Indianápolis, Lincoln, Los Angeles, } \\
\text { Miami, Nashville, Olimpia, Provo, Raleigh, Sacramento, Salt Lake } \\
\text { City, San Francisco, Seattle, Storrs y Tallahasee. }\end{array}$ \\
\hline FILIPINAS & Manila \\
\hline IRLANDA & Dublin \\
\hline PAISES BAJOS & Amsterdam \\
\hline MARRUECOS & Rabat, Agadir, Casablanca \\
\hline NUEVA ZELANDA & Auckland \\
\hline REINO UNIDO & Edimburgo, Manchester \\
\hline SUECIA & Estocolmo \\
\hline TÚNEZ & Túnez \\
\hline
\end{tabular}

Fuente: Elaboración propia

d) Direcciones de Programas

El Ministerio de Educación, Cultura y Deporte está también presente en el exterior a través de una serie de Dirección de Programas en los cuales se lleva a cabo una labor educativa en áreas determinadas:

* Guinea Ecuatorial: Es el único país africano miembro de Naciones Unidas que tiene el español como lengua oficial. Cabe destacar la labor de defensa del español como lengua oficial y de trabajo en la Unión Africana y en otros ámbitos multilaterales que Guinea Ecuatorial lleva a cabo.

España mantiene relaciones diplomáticas con Guinea Ecuatorial desde su independencia en 1968, aunque fueron brevemente interrumpidas en el último período del Gobierno de Macías Nguema. A partir de 1979, España realizó un esfuerzo considerable en la cooperación al desarrollo, en lo que constituyeron las primicias de la posterior política de cooperación. Los vínculos históricos, culturales y humanos entre ambos países marcan nuestras relaciones. Entre otros ámbitos de colaboración, España y Guinea Ecuatorial promueven activa y conjuntamente la utilización del español en la Unión Africana.

La Cooperación Española se ha centrado tradicionalmente en tres pilares básicos, educación, sanidad y cultura, y se encuentra profundamente imbricada en el tejido social ecuatoguineano. A lo largo de los años, Guinea Ecuatorial ha sido uno de los principales receptores netos de la Cooperación Española.

Tradicionalmente los sectores de concentración de la Cooperación Española han sido Educación y Salud, con el objetivo de atender las necesidades sociales básicas de la población ecuatoguineana en todo el territorio nacional, sectores que se mantendrán, en principio, en la XII Comisión Mixta, junto con el apoyo a acciones en Gobernabilidad democrática y fortalecimiento institucional. Por último, debe destacarse también la cooperación cultural a través de los Centros Culturales de Malabo y Bata. 
Así, y a la luz del IV Plan Director de la Cooperación Española, se ha optado por una serie de orientaciones como la consolidación de procesos democráticos y del Estado de Derecho, la reducción de desigualdades, el reforzamiento de los servicios sociales básicos, la provisión de bienes públicos globales o la promoción de oportunidades económicas para los más desfavorecidos. Las líneas de trabajo principales siguen siendo la educación, la sanidad y la cultura, aunque se contemplan otras como el refuerzo institucional o la formación del sector público.

* Turquía: La oficina del Ministerio de Educación, Cultura y Deporte en Turquía es denominada Dirección de Programas Educativos y sus objetivos principales son:

- acercar la lengua y cultura españolas a profesores, hispanistas, estudiantes y en general a todos aquellas personas interesadas en España y el mundo hispánico.

- colaborar con todos los profesionales de la enseñanza de español y, en general con los estudiantes y estudiosos de esta lengua en Turquía, para que colaboren con la oficina activamente y utilicen los recursos que esta Dirección de Programas Educativos pone a su disposición.

- Cabe destacar entre otras actividades promovidas desde esta oficina las siguientes: becas de formación MECD; jornadas de Formación ELE en Antalya; Talleres sobre el uso de la música en la clase de ELE; Encuentro de alumnado Erasmus en Ankara; Festival de cine español en Ankara; Taller sobre herramientas para la creación de actividades multimedia; Curso sobre recursos y estrategias para la clase de ELE o la celebración del Día de la Lengua Española en Ankara.

Esta diversidad de actividades demuestran la amplitud que la acción educativa española pretende ofertar y proponer en este país. La apertura internacional del Ministerio de Educación, Cultura y Deporte implica una adaptación a las necesidades de cada sociedad en la que se instala.

\subsection{Centros de Recursos}

La principal misión de la red de Centros de Recursos, dependientes de las Consejerías de Educación en el exterior, es apoyar la enseñanza de la lengua y la cultura españolas. En estos centros los profesores y el público en general tienen a su disposición, mediante un servicio de préstamo, colecciones de libros y materiales didácticos, tanto impresos como audiovisuales.

Otra de las funciones de los Centros de Recursos es la organización de talleres, seminarios y grupos de trabajo sobre temas relacionados con la enseñanza del español en todas sus vertientes.

Tabla 3. Relación de Centros de Recursos en el exterior

\begin{tabular}{|l|l|}
\hline \multicolumn{1}{|c|}{ PAÍS } & \multicolumn{1}{c|}{ UBICACIÓN } \\
\hline ARGENTINA & Centros de Recursos de Buenos Aires \\
\hline AUSTRALIA & Centros de Recursos de Camberra y de Melbourne \\
\hline BELGICA & Centros de Recursos de Bruselas \\
\hline
\end{tabular}




\begin{tabular}{|c|c|}
\hline BRASIL & Centros de Recursos de Brasilia y de Salvador de Bahía \\
\hline BULGARIA & Centros de Recursos de Sofía \\
\hline CANADA & Centros de Recursos de Edmonton, de Montreal y de Toronto. \\
\hline CHINA & Centros de Recursos de Beijing \\
\hline ESLOVAQUIA & Centros de Recursos de Bratislava \\
\hline $\begin{array}{l}\text { ESTADOS } \\
\text { UNIDOS }\end{array}$ & $\begin{array}{l}\text { Centro de Recursos Baton Rouge, Boston, Houston, Indianápolis, } \\
\text { Kenessaw, Lincoln, Los Ángeles, Miami, Provo, Seattle y Storrs }\end{array}$ \\
\hline FILIPINAS & Centro de Recursos de Manila \\
\hline FRANCIA & Centros de Recursos de París \\
\hline HUNGRIA & Centros de Recursos de Budapest \\
\hline ITALIA & Centros de Recursos de Roma \\
\hline LUXEMBURGO & Centros de Recursos de Luxemburgo \\
\hline MARRUECOS & Centros de Recursos de Marruecos \\
\hline MEXICO & Centros de Recursos de México D.F. \\
\hline PAISES BAJOS & Centros de Recursos de Ámsterdam \\
\hline POLONIA & Centros de Recursos deVarsovia \\
\hline PORTUGAL & Centros de Recursos de Lisboa \\
\hline REINO UNIDO & Centros de Recursos de Londres, Manchester y Edimburgo. \\
\hline $\begin{array}{l}\text { REPÚBLICA } \\
\text { CHECA }\end{array}$ & Centros de Recursos de Praga \\
\hline RUMANÍA & Centros de Recursos de Bucarest \\
\hline RUSIA & Centros de Recursos de Moscú \\
\hline SUIZA & Centros de Recursos de Berna \\
\hline TUNEZ & Centros de Recursos de Túnez \\
\hline TURQUÍA & Centros de Recursos de Ankara \\
\hline
\end{tabular}

Fuente: Elaboración propia 


\subsection{Centros Docentes}

Tabla 4. Tipos de centros y programas del MECD en el exterior.

\begin{tabular}{l}
\hline Centros de Titularidad del Estado Español \\
Centros de Titularidad Mixta \\
Centros de Convenio \\
Agrupaciones de Lengua y Cultura españolas \\
Secciones españolas en centros de titularidad de otros estados \\
Secciones bilingues en Europa Central y Oriental, China y Turquía \\
Escuelas Europeas \\
ISAS (International Spanisch Academies) \\
\hline
\end{tabular}

Fuente: Elaboración propia.

Otras de las estructuras que el Ministerio de Educación, Cultura y Deporte dispone en el exterior son los centros docentes. A continuación se va a concretar qué función desempeñan cada uno de ellos.

\subsubsection{Centros de Titularidad del Estado Español}

En estos centros se imparten enseñanzas regladas del sistema educativo español de nivel no universitario, dirigidas tanto a españoles como extranjeros, y adecuándolas a las necesidades específicas del alumnado y a las exigencias del entorno socio-cultural. Su creación corresponde al Gobierno, mediante Real Decreto aprobado en Consejo de Ministros, a propuesta conjunta de los Ministros de Educación y de Asuntos Exteriores. Estos centros deberán tener una denominación específica e inscribirse en el Registro público existente al efecto en el Ministerio de Educación, Cultura y Deporte.

Estos centros docentes, están sujetos al principio de unidad de acción del Estado en el exterior y sometidos a la dependencia del Jefe de la Misión Diplomática a efectos de su coordinación. Podrán ser centros específicos de un determinado nivel o etapa del sistema educativo español o centros integrados en los que se impartan enseñanzas de diferentes niveles o etapas.

\subsubsection{Centros de Titularidad Mixta}

Con objeto de propiciar la proyección de la educación y de la cultura española, la Administración establece convenios con Administraciones extranjeras o personas físicas o jurídicas de nacionalidad española o extranjera, para la creación de centros de titularidad mixta a través de fundaciones o de sociedades, reconocidas legalmente en los países respectivos.

Los convenios deben garantizar que la representación institucional española sea mayoritaria en las respectivas fundaciones o sociedades y en los órganos rectores de los mismos, que quedarán sometidos al principio de unidad de acción en el exterior. 
Estos centros son dirigidos por funcionarios españoles nombrados por el Ministerio de Educación, Cultura y Deporte, tienen un régimen económico autónomo y se rigen por las normas de organización y funcionamiento que establecen los convenios correspondientes y los respectivos reglamentos de régimen interior. Pueden impartir enseñanzas del sistema español o de los sistemas educativos de los países respectivos, con un componente adecuado, en este supuesto, de lengua y cultura españolas.

\subsubsection{Centros de Convenio}

Con el fin de ampliar la oferta educativa española en otros países, el Ministerio de Educación, Cultura y Deporte ha abierto la posibilidad de suscribir convenios de colaboración con Instituciones o Fundaciones situadas fuera de nuestro país al amparo de lo establecido en la Orden ECI/1711/2005, de 23 de mayo (BOE de 10 de junio), que fija las bases para la suscripción de convenios con las instituciones a que se refiere el artículo 7.1. d) del Real Decreto 1027/1993 de 25 de Junio, que regula la Acción Educativa en el Exterior.

Los centros que firman un convenio suelen gozar de un reconocido prestigio académico en su país. Con ello se pretende que los centros de convenio se conviertan en centros de referencia educativa, desde los que se dé a conocer, a otras instituciones docentes del país y a cuantos profesores que puedan estar interesados toda la información relacionada con los cursos de formación del profesorado, con los Programas de Nuevas Tecnologías aplicadas a la educación, con las novedades editoriales relacionadas con materiales didácticos y publicaciones, etc.

\subsubsection{Agrupaciones de Lengua y Cultura española}

Dentro de otra modalidad, la Administración española promueve, a través de convenios o acuerdos internacionales o de las fórmulas de colaboración que resulten pertinentes, la integración, en los sistemas educativos de los distintos países, de enseñanzas de lengua y cultura españolas dirigidas al alumnado español escolarizado en dichos sistemas.

Las enseñanzas de la lengua y cultura españolas van dirigidas a alumnos españoles escolarizados en niveles no universitarios de los sistemas educativos de otros países. Se imparten fuera del horario escolar y se articulan en 5 niveles (del A1 al C1) correspondientes al Marco Común Europeo de Referencia, comprendiendo un total de 10 años.

\subsubsection{Secciones españolas en centros de Titularidad de otros Estados.}

Las secciones españolas se crean en centros docentes no universitarios de otros Estados con el fin de ofrecer enseñanzas en español integradas en el sistema educativo del país. Desarrollan currículos mixtos que incluyen asignaturas impartidas en la lengua del país y en español; entre estas últimas figuran enseñanzas de lengua y literatura española y de geografía e historia. En algunas secciones se imparten también en español otras materias. Se rigen por las normas internas de organización y funcionamiento de los centros de los que forman parte y por las acordadas bilateralmente con las autoridades respectivas al amparo de los acuerdos o convenios correspondientes. Los estudios cursados tienen validez en los sistemas educativos de los países donde están implantadas y su reconocimiento en el sistema educativo español se obtiene mediante la posterior convalidación u homologación, aunque en algunos casos se otorga también la titulación española (Francia y Miami). 


\subsubsection{Secciones bilingues en Europa central y oriental, China y Turquía.}

Con objeto de ampliar las posibilidades de recibir educación española en el contexto de experiencias educativas interculturales, la Administración española puede colaborar en el establecimiento de secciones españolas o secciones bilingües, en centros de titularidad de otros Estados o de Organismos internacionales, en los que se impartan enseñanzas de niveles no universitarios con validez en otros sistemas educativos.

El interés por la oferta cultural y educativa española y en especial por el estudio del español como idioma extranjero, ha crecido de forma considerable en toda el área de Europa central y oriental durante los últimos años. El Ministerio de Educación, Cultura y Deporte consciente de la situación ha tratado de satisfacer este interés, desarrollando los programas de difusión del español como lengua extranjera y promoviendo cauces permanentes de difusión de nuestra cultura.

\subsubsection{Escuelas Europeas}

Las Escuelas Europeas son centros educativos creados conjuntamente por los gobiernos de los Estados miembros de la Unión Europea con el fin de ofrecer una educación dentro de una Europa multicultural y plurilingüe, cuyos destinatarios son (de forma prioritaria) los hijos de los funcionarios de las instituciones europeas. Las escuelas gozan de un estatuto jurídico como centro de enseñanza pública en los respectivos países.

El principio fundamental de estas Escuelas es apoyar la construcción de una identidad europea, permitiendo a sus alumnos reafirmar su propia identidad cultural, así como su identidad como ciudadanos europeos. Con el fin de proporcionar una auténtica educación multicultural se pone un énfasis especial en el aprendizaje, compresión y uso de lenguas extranjeras.

Las Escuelas Europeas ofrecen enseñanzas de educación infantil (alumnos a partir de los 4 años), primaria (cinco cursos, de los 6 a los 11 años) y secundaria (siete cursos, de los 11 a los 18 años). En las Escuelas Europeas el alumnado se divide por secciones lingüísticas, de forma que un alto porcentaje de las enseñanzas se imparte en la lengua de la sección. Al finalizar la educación secundaria, se obtiene el Bachillerato Europeo que goza del mismo estatuto que los títulos y pruebas nacionales oficiales de los Estados miembros de la UE.

\subsubsection{ISAS (International Spanich Academies)}

Las International Spanish Academies (ISAs) son centros educativos de cualquiera de los niveles de enseñanza no universitaria ("PreK, Elementary, Middle, High School"), de reconocido prestigio a nivel académico, que siguen (con la totalidad o una parte de su alumnado) un programa de curriculum integrado de lengua y contenidos en los que el español se utiliza como lengua vehicular.

Estos centros firman un convenio de colaboración con el Ministerio de Educación, Cultura y Deporte de España y su programa se enmarca en las actividades de cooperación entre la Consejería de Educación en Estados Unidos y Canadá y las autoridades educativas norteamericanas.

El programa comenzó como experiencia piloto en los Estados de Illinois, Indiana, Florida y en la provincia de Alberta en el año 2000, como iniciativa de la Consejería de 
Educación para aprovechar el enorme crecimiento de los programas de enseñanza aprendizaje que integran lengua y contenidos curriculares.

\section{La convivencia y la interculturalidad en el entorno educativo}

Tras acercarse a la acción educativa que el Ministerio de Educación, Ciencia y Deporte realiza fuera de sus fronteras, se va iniciar una reflexión sobre el binomio interculturalidad y convivencia que se desarrolla en los centros docentes en concreto.

Entre las finalidades de estos centros debe estar la integración y la convivencia del alumnado de diversas culturas y nacionalidades en el marco escolar (independiente de la modalidad educativa); pero no es únicamente en su pedagogía donde deben resaltarse valores orientados a la aceptación pasiva de otra cultura, sino más bien en su reconocimiento activo donde la implicación de toda la comunidad educativa y en todo el curriculum, permita percibir el enfoque intercultural que transfiere toda su estructura organizativa y operativa.

Los centros docentes en el exterior son establecimientos donde la convivencia de las diversas naciones invita al conocimiento mutuo y al intercambio entre los espacios culturales. En él parece esencial que el alumnado pueda sacar provecho de esta posición excepcional durante el tiempo de su escolaridad. En programas como secciones internacionales, Escuelas Europeas, ISAS o secciones bilingues, la interacción de diversas culturas está presente en todo el proceso de enseñanza/aprendizaje del centro en el que se desarrolla. Integrarse en un sistema educativo diferente o compartir o compartir el proceso con diferentes sistemas educativos es lo que acentúa esa realidad de convivencia entre culturas.

En algunos casos, podemos encontrarnos con centros con una importante diversidad cultural aportada por el personal docente, las familias, el alumnado, etc., y por otro lado, ante una realidad multicultural amplia y compleja, en la que participen culturas bien diferentes entre sí y con orígenes dispares como la japonesa, la americana o la rusa. Ejemplo de esta son algunas secciones internacionales de Francia donde la convivencia intercultural de catorce secciones marca el desarrollo de gran parte de su proceso educativo. En las Escuelas Europeas, la interacción y convivencia de diferentes culturas es similar y las actividades conjuntas que se plantean contribuyen a ese respeto y tolerancia en toda la comunidad educativa que la forma.

Se puede decir que, en estos casos, la interculturalidad supone recoger la multiculturalidad que desprende la estructura del centro para transformarla en un encuentro enriquecedor y formativo entre el alumnado, pero también entre las familias y el contexto social. Requiere una formación que transforme los estereotipos negativos y los prejuicios en actitudes positivas hacia el encuentro y la fusión con otras culturas. Dicha formación la aporta el MECD con su personal desplazado y con los objetivos y fines que se han reflejado en puntos anteriores.

En relación a los diferentes tipos de centros docentes que forman parte de la acción educativa en el exterior, y con el objetivo de alcanzar una apropiada convivencia y una educación intercultural en ellos, se debería lograr : una buena participation de las familias; Impulsar y difundir el uso de la lengua dentro de las actividades ordinarias; Cuando se combinen la lengua materna y la del país de acogida no se producirán problemas de comunicación porque el alumnado percibe la enseñanza de la 2 a lengua como algo menos distante de su cultura y entorno social y de esta manera,el alumnado procedente de otro país se siente más integrado; Difundir materiales curriculares y experiencia didácticas realizadas 
en los centros; ofertar actividades diversas como ciclos de cine, actividades en asociaciones, jornadas para la formación conjunta de toda la diversidad del profesorado existente en el centro, etc. Son instituciones educativas perceptoras de la convivencia entre diferentes culturas y su papel es determinante para el desarrollo de la sociedad. Su objetivo, se puede decir que, es desarrollar el potencial del alumnado gracias a la transmisión del saber y al desarrollo de competencias, de actitudes y de valores que le proporcionarán los medios necesarios para vivir en una sociedad multicultural como la actual.

Además, para reforzar la aproximación que los centros en el exterior tienen respecto a la convivencia intercultural, estos consideran que se deben valorar los aspectos positivos de otras culturas; se potencie la igualdad de oportunidades académicas en todo el alumnado; se introduzcan elementos culturales en sus aulas; se descubran las semejanzas culturales y se mejore el autoconcepto personal, cultural y académico del alumnado.

Si lo que se desea es favorecer la convivencia de diferentes grupos culturales en un mismo medio mediante la transmisión de la cultura plural, es necesario atenuar todas las culturas y adoptar una actitud de hendimiento cultural. El MECD, en sus diferentes programas educativos, participa en ello; potencia la convivencia entre alumnado culturalmente diferentes, dentro y fuera de la escuela y la convivencia cultural es por tanto natural en todas las edades.

\section{Conclusiones}

La diversidad de culturas es un hecho bien latente en nuestras comunidades y por ello, desde la perspectiva internacional de este artículo que se presenta, se ha considerado relevante adentrarse en el conocimiento de esta acción educativa que se puedan estar ofreciendo desde un plano de apertura mundial.

Más allá de los centros docentes que cada Estado reconoce en su sistema educativo, en nuestros días se están adoptando cada vez mayores iniciativas extranjeras dentro del fenómeno de la globalización. Salir de las fronteras regionales o nacionales supone abrirse a la comunidad educativa internacional y dar oportunidad a nuevas ideas constructivas de mejora y prosperidad. Este fenómeno se produce gracias a la cooperación y el interés internacional por acercar culturas y lenguas.

La dimensión internacional de actuación de los diferentes Estado ha cobrado en los últimos tiempos un protagonismo sin precedentes. La razón de ello es que en un mundo globalizado e interdependiente como el actual, la forma de conducir la política exterior de un Estado incide de manera directa e inmediata en la vida e intereses de sus ciudadanos.

Por esta razón, se observa que cuando se habla de educación estamos hablando de un acto que conlleva un qué, un para qué y un por qué basado en la convivencia, el ser humano y la cultura.

Ello quiere decir que si todo proceso de convivencia humana (presente en cualquier entorno educativo) se basa en el respeto a la relación recíproca de valores, podemos deducir que cuando hablamos de culturas se apela a la valoración del bagaje ético de varias culturas, en donde la interrelación se basa justamente en el respeto a estos valores.

No es una tarea fácil promover una convivencia intercultural dentro de los centros educativos pero sí se debe apostar por el respeto y la homologación de las culturas así como 
valorar la diversidad cultural como una fuente de enriquecimiento. Incidir, como señala Viana-Orta (2014), en la importancia de los acuerdos o pactos sociales a favor de una cultura de paz y de una convivencia pacífica en los centros educativos. De esta manera se podría empezar a percibir la interculturalidad como un código necesario para la convivencia.

Con este artículo se pretende dar a conocer la profundidad de la acción educativa del MECD en el exterior en lo que se refiere al ámbito de los valores, la convivencia y las relaciones interculturales dentro de sus programas educativos. Por ello, apuesta hacia la convivencia de varias culturas evitando así el prefijo "pluri" que puede hacer referencia a una pluralidad de culturas (Sánchez Lázaro, 2009) y podría darse a entender que se realiza en sus programas y centros. Al tratarse de prácticas educativas, se considera conveniente finalizar este documento desde el mismo punto de partida con el que se inició, la Ley Orgánica 2/2006, de 3 de mayo, de Educación que en su preámbulo establece uno de los fines que se justificaba en el apartado 1 al vincular anteriores trabajos realizados en el ámbito de los valores y la educación intercultural:

"Para la sociedad, la educación es el medio de transmitir y, al mismo tiempo, de renovar la cultura y el acervo de conocimientos y valores que la sustentan, de extraer las máximas posibilidades de sus fuentes de riqueza, de fomentar la convivencia democrática y el respeto a las diferencias individuales, de promover la solidaridad y evitar la discriminación, con el objetivo fundamental de lograr la necesaria cohesión social".

\section{Referencias bibliográficas}

Funciones de la Subdirección General de Cooperación Internacional y Promoción Exterior Educativa. Recuperado de: https://www.mecd.gob.es/ministeriomecd/organizacion/organigrama/ministro/secretaria-estado-educacion/n/dgplanificacion-gestion/sg-coop-int-promocion-ext-edu.html

García, A. y Escarbajal, A.(2009). Pluralismo sociocultural, educación e interculturalidad (23-42). Badajoz: @becedario.

Creación del Instituto Cervantes (Ley 7/1991 de 21 de marzo). Boletín Oficial del Estado, $\mathrm{n}^{\circ} 70,1991,22$ de marzo.

Ley orgánica de educación (LOE) (Ley Orgánica 2/2006, de 3 de mayo). Recuperado de: https://www.boe.es/buscar/pdf/2006/BOE-A-2006-7899-consolidado.pdf

Ley Orgánica General del Sistema Educativo (LOGSE) (Key Orgánica 1/1990, de 3 de octubre). Boletín Oficial del Estado, n²38, 1990, 4 de Octobre.

Martínez Usarralde, M.J. (Ed.) (2010). Y para muestra... Políticas educativas de inmigración y modelos de escuela que practican la interculturalidad. Valencia: Departamento de Educación Comparada e Historia de la Educación de la Universitat de València.

Convivencia Escolar. Ministerio de Educación, Ciencia y Deporte. Recuperado de: https://www.mecd.gob.es/educacion/mc/convivencia-escolar/inicio.html

Real Decreto 1027/1993, de 25 de junio, por el que se regula la acción educativa en el exterior. Boletín Oficial del Estado, n¹87, 1993, 6 de agosto. 
Sánchez Lázaro, A.M. (2009). Multiculturalidad e interculturalidad. En García, A. y Escarbajal, A. Pluralismo sociocultural, educación e interculturalidad. Badajoz: @ becedario.

Viana-Orta, M.I. (2014). La medicación escolar en los planes y programas institucionales de convivencia en España. Revista Complutense de Educación, 25(2), 271-291. 